\title{
Twisted particles as a new tool to study micro world phenomena and processes ${ }^{a}$
}

\author{
Anna Mayorova ${ }^{1 *}$ \\ ${ }^{1}$ Peter the Great Saint Petersburg Polytechnic University, Centre for Advanced Studies, 195251 \\ Polytechnicheskaya st. 29, Russian Federation
}

\begin{abstract}
Twisted or vortex particles are a new powerful tool to study atomic and molecular processes as well as phenomena that occur at the level of nano-objects. The main feature of such particles is that they carry a non-zero projection of orbital angular momentum along the beam propagation direction. The process of twisted electron scattering from diatomic molecule targets has been studied in this paper for the first time. The Yukawa potential is selected as a model potential. Numerical calculations are carried out for the case of scattering from a hydrogen molecule $\mathrm{H}_{2}$.
\end{abstract}

\section{Introduction}

Over the last two decades, conceptually new tools became available to be used in studying such micro world phenomena as twisted, or vortex, particles. Twisted photons were theoretically predicted [1] and experimentally implemented [2] in the end of the 20th century. In 2007, the properties of twisted electrons were described in detail, the behaviour of twisted electrons in external fields was studied, and a number of experiments to obtain twisted electrons was proposed in the publication [3]. The publication gave rise to a series of experimental work on obtaining twisted electron beams. Today, it is possible to produce twisted electron beams with an energy of about a few hundreds $\mathrm{keV}$ and projections of the angular momentum $m \hbar \sim 100 \hbar$. Moreover, first steps to obtain twisted neutron beams [4] and even twisted matter waves [5] have recently been taken. Twisted particles serve as a powerful research tool in various fields of modern physics. For example, in quantum information theory, twisted photons may be used to transmit data since the information capacity of twisted states is significantly higher than that one of preset helicity states, as well as to multiplex data flows. Furthermore, twisted photons may be used to manipulate individual atoms, to carry out fundamental verifications of the STR and the GTR, and to analyse fundamental processes of the interaction of light with matter. Twisted light in optical traps enables not only to trap a nano-object (e.g. a biological structure or the BoseEinstein condensate), but also to rotate it. Twisted electrons may be used to study structural and electronic properties of a matter or magnetic properties of surfaces and solid bodies.

\footnotetext{
a This study was supported by the Foundation for the Advancement of Theoretical Physics "BASIS" and the Ministry of Education and Science of the Russian Federation (grant no. 3.1463.2017/4.6)

*Corresponding author: majorova av@spbstu.ru
} 


\section{Theory and results}

The state of a twisted particle is described by a wave; the wave front represents a helical curve. Therewith, there is a relationship between the wave phase and the azimuth angle $\varphi$ (Fig. 1 a). The intensity profile of such wave in the plane perpendicular to its direction of propagation is a set of concentric rings with a central null-intensity spot (vortex) (Fig.1 b). Moreover, the state of a twisted particle in momentum space may be described by a superposition of plane waves with the wave vector $\mathbf{k}=\left(\mathbf{k}_{\perp}, k_{\mathrm{z}}\right)$ which lays on the surface of a cone with the opening angle $\theta_{\mathrm{k}}$ (Fig. $1 \mathrm{c}$ ). When the opening angle $\theta_{\mathrm{k}}$ becomes zero, the twisted wave turns into a common plane wave.

a)

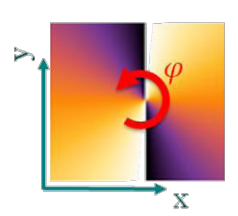

b)

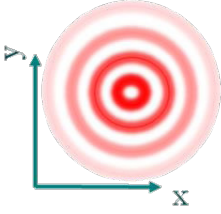

c)

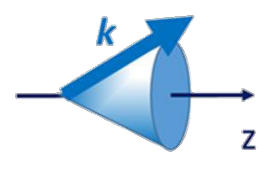

Fig. 1. a) twisted wave phase, b) twisted wave intensity, c) wave vector $\mathbf{k}$ of the twisted wave in momentum space.

Twisted waves may be described by what is known as the Bessel wave. The Bessel wave is a stationary state having a definite energy $\omega$, a longitudinal momentum $k_{\mathrm{z}}$, helicity $\lambda$, and an orbital angular momentum projection $m$ :

$$
\Psi_{t w}(\mathbf{r})=\int \frac{d^{2} \mathbf{k}_{\perp}}{(2 \pi)^{2}} a_{æ m}\left(\mathbf{k}_{\perp}\right) e^{i \mathbf{k r}}
$$

where

$$
a_{æ m}\left(\mathbf{k}_{\perp}\right)=(-i)^{m} e^{i m \varphi} \delta\left(\left|\mathbf{k}_{\perp}\right|-æ\right) \frac{1}{æ}
$$

A series of studies of various processes of collisions with twisted electrons was carried out over the last decade. However, in all these studies, twisted electron beam interacted with either a single counter-propagating beam (Compton scattering [6], electron-electron scattering [7]) or a target consisting of atoms (radiative recombination [8-9], Mott scattering [10]). The process of twisted electron scattering on target molecules has been studied herein for the first time. It is well known that when colliding with a molecule, highspeed electrons generally interact with nuclei and electrons of the inner shell of atoms which form the given molecule. Therewith, molecular centres play the role similar to that one of optical slit in light scattering, since they are localized in space and distinctly separated from each other. As a result of the interaction of electrons with the molecule, a strong interference occurs in the differential cross-section. The use in this experiment of such twisted electrons instead of the usual electrons described by a plane wave leads to a number of peculiarities. Three possible scenarios are studied in this paper: (I) scattering from one molecule which centre of gravity lays on the beam axis (Fig. 2), (II) scattering from a molecule with one of its atoms laying on the beam axis (Fig. 3), and (III) scattering from a macroscopic target consisting of a plurality of molecules (Fig. 4). 


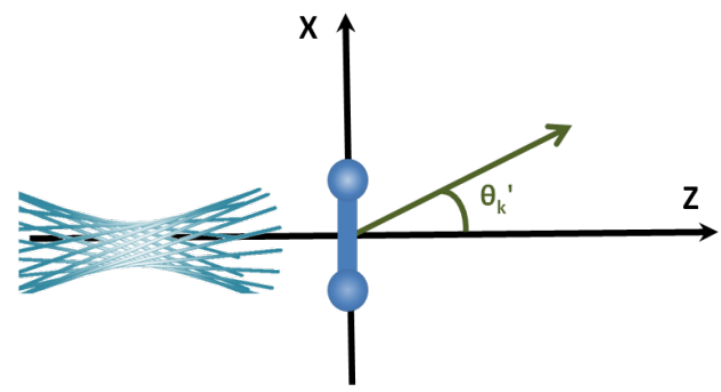

Fig. 2. Scenario (I) geometry: the centre of gravity of the molecule lays on the beam axis.

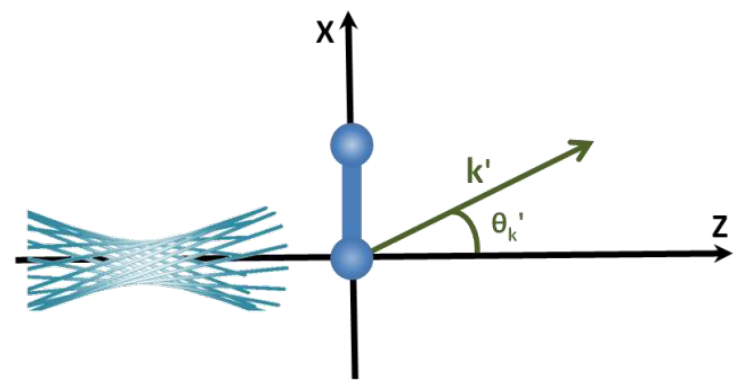

Fig. 3. Scenario (II) geometry: one of the atoms in the molecule lays on the beam axis.

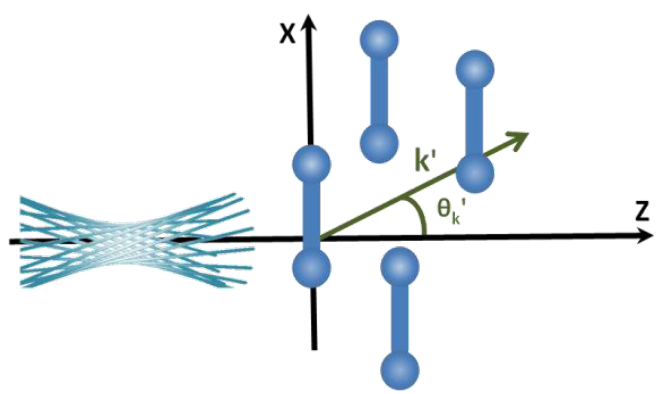

Fig. 4. Scenario (III) geometry: macroscopic target.

Numerical calculations of angular distributions and cross-sections for different scenarios have been carried out for the hydrogen molecule $\mathrm{H}_{2}$. This study uses the relativistic system of units $\hbar=c=m_{e}=1$. The Yukawa potential is selected as a model potential:

$$
V(r)=A \frac{e^{-r / a}}{r}
$$

All calculations have been carried out in the first Born approximation. The amplitude of twisted wave scattering from two centres takes the following form:

$$
f_{2}^{t w}\left(\mathbf{k}, \mathbf{k}^{\prime}\right)=(-i)^{m} e^{i \mathbf{k}_{\perp} \mathbf{b}} \int \frac{d^{2} \mathbf{k}_{\perp}}{(2 \pi)^{2}} e^{i m \varphi-i \mathbf{k}_{\perp} \mathbf{b}_{\mathbf{0}}} \delta\left(\left|\mathbf{k}_{\perp}\right|-æ\right) \frac{1}{æ}\left(1+e^{i \mathbf{q b} \mathbf{b}}\right) f_{1}\left(\mathbf{k}, \mathbf{k}^{\prime}\right)
$$

where

$$
f_{1}\left(\mathbf{k}, \mathbf{k}^{\prime}\right)=-2 \int_{0}^{\infty} r V(r) \sin q r d r
$$


is the amplitude of plane wave scattering from one centre, $\mathbf{b}$ is the atomic spacing in the molecule, $\mathbf{b}_{\mathbf{0}}$ is the distance between the beam axis and the nearest atom, $\mathbf{q}=\mathbf{k}^{\prime}-\mathbf{k}$ is the momentum transferred. The calculation results are shown in Fig. 5 to 7. As is clear from Fig. 5, when the molecule centre is on the beam axis (Scenario (I)), there is a strong dependence from the projection of angular momentum $m$, and the maximums and minimums of the angular distribution are interchanged. As the energy increases, the number of minimums (and maximums) grows. This scenario is a quantum analogue of the classical Young's double-slit experiment. Scenario (II) corresponds to a classical experiment when one of the slits is closed. The strong dependence from the projection of angular momentum $m$ is still observed (Fig. 6). However, the interference disappears, depending on the projection and the opening angle. Scenario (III) corresponds to scattering from a macroscopic target. In this case, the scattering cross-section no longer depends on the angular momentum projection. As one should expect, the contributions of multiple slits are non-coherently summed, which results in disappearance of the interference pattern (Fig. 7).
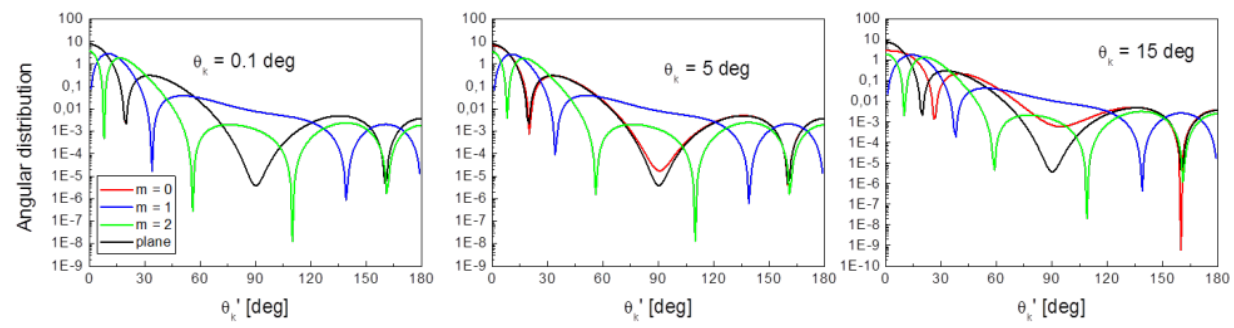

Fig. 5. Results of numerical calculations for Scenario (I). Angular distribution in case of scattering of twisted electrons with $\mathrm{E}=300 \mathrm{eV}$ from the hydrogen molecule $\mathrm{H}_{2}$.
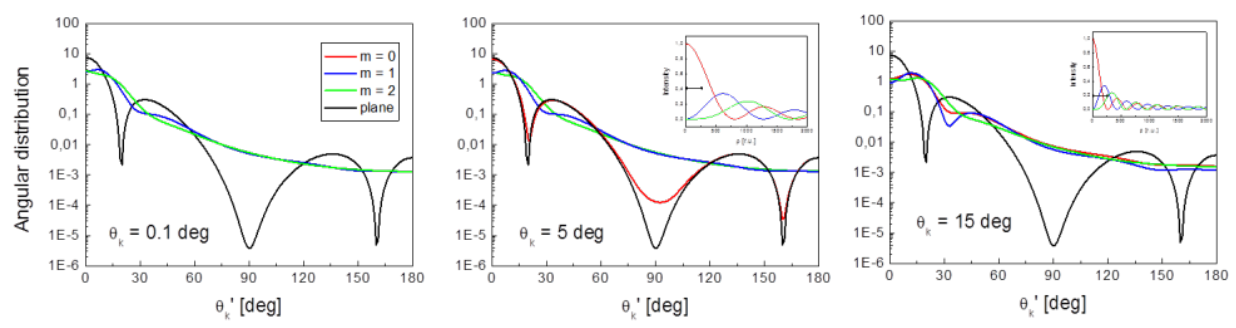

Fig. 6. Results of numerical calculations for Scenario (II). Angular distribution in case of scattering of twisted electrons with $\mathrm{E}=300 \mathrm{eV}$ from the hydrogen molecule $\mathrm{H}_{2}$.
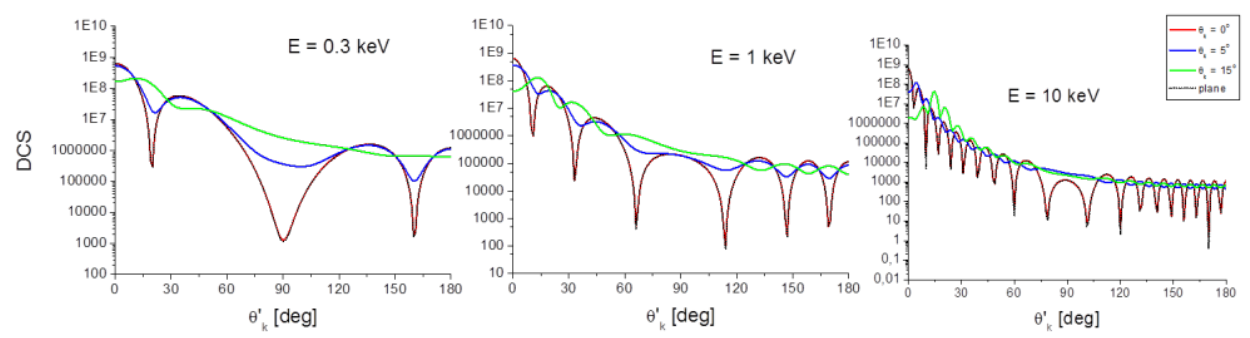

Fig. 7. Results of numerical calculations for Scenario (III). Scattering of twisted electrons from a macroscopic target. 


\section{Conclusion}

The theoretical study of the process of twisted electron beam scattering from a diatomic molecule have been carried out for different geometries of the process for the first time. The numerical calculations are given for the hydrogen molecule. Studying the interaction of twisted electrons with target molecules is an important step to studying such fundamental phenomenon of living systems as chirality.

\section{References}

1. L. Allen, M.W. Beijersbergen, R.J.C. Spreeuw, J.P. Woerdman, Phys. Rev. A, 45 (1992)

2. H. He, M.E.J. Friese, N.R. Heckenberg, H. Rubinsztein-Dunlop, Phys. Rev. Lett. 75 (1995)

3. K.Y. Bliokh, Y.P. Bliokh, S. Savel'ev, F. Nori, Phys. Rev. Lett., 99 (2007)

4. C.W. Clark, R. Barankov, M.G. Huber, M. Arif, D.G. Cory, D.A. Pushin, Nature, 525 (2015)

5. C. Ryu, K.C. Henderson, M.G. Boshier, New Jour. of Phys, 16 (2014)

6. D. Seipt, A. Surzhykov, S. Fritzsche, Phys. Rev. A, 90 (2014)

7. I.P. Ivanov, D. Seipt, A. Surzhykov, S. Fritzsche, Phys. Rev. D, 94 (2016)

8. O. Matula, A.G. Hayrapetyan, V.G. Serbo, A. Surzhykov, S. Fritzsche, New J. Phys., 16 (2014)

9. V.A. Zaytsev, V.G. Serbo, V.M. Shabaev, Phys. Rev. A, 95 (2017)

10. V. Serbo, I.P. Ivanov, S. Fritzsche, D. Seipt, A. Surzhykov, Phys. Rev. A, 92 (2015) 